\title{
Local properties of strengthened ordered directional and other forms of monotonicity
}

\author{
Mikel Sesma-Sara, Laura De Miguel, Radko Mesiar, Javier Fernandez and \\ Humberto Bustince
}

\begin{abstract}
In this study we discuss some of the recent generalized forms of monotonicity, introduced in the attempt of relaxing the monotonicity condition of aggregation functions. Specifically, we deal with weak, directional, ordered directional and strengthened ordered directional monotonicity. We present some of the most relevant properties of the functions that satisfy each of these monotonicity conditions and, using the concept of pointwise directional monotonicity, we carry out a local study of the discussed relaxations of monotonicity. This local study enables to highlight the differences between each notion of monotonicity. We illustrate such differences with an example of a restricted equivalence function.
\end{abstract}

\section{Introduction}

A function $f:[0,1]^{n} \rightarrow[0,1]$ satisfying the conditions $f(0, \ldots, 0)=0, f(1, \ldots, 1)=$ 1 and $f\left(x_{1}, \ldots, x_{n}\right) \leq f\left(y_{1}, \ldots, y_{n}\right)$ for $x_{i} \leq y_{i} \in[0,1]^{n}$, for all $i \in\{1, \ldots, n\}$, is said to be an aggregation function [2, 12]. Aggregation functions are aimed at fusing data by representing with a single value the information coming from $n$ values.

Mikel Sesma-Sara

Public University of Navarra, Pamplona, Spain e-mail:mikel. sesma@unavarra.es

Laura De Miguel

Public University of Navarra, Pamplona, Spain e-mail: laura.demigueleunavarra.es

Radko Mesiar

Slovak University of Technology, Bratislava, Slovakia e-mail: mesiar@math.sk

Javier Fernandez

Public University of Navarra, Pamplona, Spain

e-mail: fcojavier.fernandez@unavarra.es

Humberto Bustince

Public University of Navarra, Pamplona, Spain e-mail: bustince@unavarra.es 
There exist a great number of works that study aggregation functions, both from the theoretical [1, 7, 10] and the practical [9, 16, 21] perspectives.

On occasion, the monotonicity condition of aggregation functions is restrictive, in the sense that it prevents some, otherwise valid, non-monotone functions to enter the framework of data fusion functions [22]. This is the case of some averaging functions like Gini and Lehmer means [3] and other mixture functions [4].

In that respect, in the literature various proposals of weaker forms of monotonicity can be found. The first one was introduced by Wilkin and Beliakov with the name of weak monotonicity [22]. A function is said to be weakly increasing if whenever the value of all its arguments increase by the same amount, the value of the function increases. An analagous manner to look at this concept is as increasingness along the ray defined by the vector $(1, \ldots, 1)$. This led to the generalization of weak monotonicity to directional monotonicity [8], which considers monotonicity along directions defined by any vector $\vec{r} \in \mathbb{R}^{n} \backslash\{\overrightarrow{0}\}$. Functions that are directionally monotone have been used in classification systems with sound results [15, 13, 14]. Weak and directional monotonicity have been extended to more general frameworks, such as lattices, intervals, intuitionistic fuzzy values, etc. in [20].

There also exist other generalizations of monotonicity, such as ordered directional (OD) monotonicity [6] and strengthened ordered directional (SOD) monotonicity [19]. These two concepts are based on directional monotonicity but the direction of increasingness varies from one point of the domain to another, depending on the relative sizes of the components of the specific point.

In this work, we study the cited notions of weak, directional, ordered directional and strengthened ordered directional monotonicity from a local point of view. All these are global properties of monotonicity. We make use of the concept of pointwise directional monotonicity, which is a local condition (studied at a specific point of the domain) of monotonicity along rays, to study the relations and differences between the mentioned relaxed forms of monotonicity. We characterize each of the forms in terms of pointwise directional monotonicity and we illustrate these findings with an example of a restricted equivalence function [5].

This paper is organized as follows. In Section 2 we fix the notation that is used in this work. In Section 3 we present the notions of weak, directional, ordered directional and strengthened ordered directional monotonicity, along with some of their most relevant properties and some examples. In Section 4, we present the definition of pointwise directional monotonicity and show how it can be use to characterize of each of the cited monotonicity forms. In Section 5 we expose some concluding remarks.

\section{Preliminaries}

Let us first set the notation for this work. We refer to points in the unit hypercube as $\mathbf{x}=\left(x_{1}, \ldots, x_{n}\right) \in[0,1]^{n}$ and we denote real vectors, which are used to express directions in the space, as $\vec{r} \in \mathbb{R}^{n}$. In particular, we use the notations $\mathbf{0}=(0, \ldots, 0)$ 
and $\mathbf{1}=(1, \ldots, 1)$. To order elements in $[0,1]^{n}$, we consider the product order $\leq_{L}$ inherited from the standard total order of $[0,1]$, i.e., for any $\mathbf{x}, \mathbf{y} \in[0,1]^{n}$ we say that $\mathbf{x} \leq_{L} \mathbf{y}$ if $x_{i} \leq y_{i}$ for all $i \in\{1, \ldots, n\}$.

Moreover, in this work, the order of the inputs of a function $f:[0,1]^{n} \rightarrow[0,1]$ has a great impact in the conditions of monotonicity that affect $f$ at the point $\mathbf{x}$ formed by these inputs. Consequently, in this work we deal with permutations of the components of points $\mathbf{x} \in[0,1]^{n}$ and we use the following notation: let $\mathscr{S}_{n}$ be the set of all permutations of $n$ elements, $\sigma \in \mathscr{S}_{n}$ and $\mathbf{x} \in[0,1]^{n}$, then $\mathbf{x}_{\sigma}$ denotes the point $\left(x_{\sigma(1)}, \ldots, x_{\sigma(n)}\right) \in[0,1]^{n}$. Similarly, given $\vec{r} \in \mathbb{R}^{n}$ we use the notation $\vec{r}_{\sigma}=\left(r_{\sigma(1)}, \ldots, r_{\sigma(n)}\right) \in \mathbb{R}^{n}$.

Once the basic notation is established, we recall the concept of an aggregation function.

Definition 1. Let $n \in \mathbb{N}$ and $A:[0,1]^{n} \rightarrow[0,1]$. We say that $A$ is an aggregation function if

1. $A(\mathbf{0})=0$ and $A(\mathbf{1})=1$;

2. $A$ is increasing with respect to all its arguments, i.e., if $\mathbf{x}, \mathbf{y} \in[0,1]^{n}$ such that $\mathbf{x} \leq_{L} \mathbf{y}$, then $A(\mathbf{x}) \leq A(\mathbf{y})$.

Note that, throughout the paper, we use the term increasing to refer to the property of non-decreasingness. We will explicitly state strict increasingness when needed.

\section{Different relaxations of monotonicity}

\subsection{Definitions}

In this section we gather some of the recently introduced relaxed forms of monotonicity. Among those that are object of study in this work, weak monotonicity was introduced first [22].

Definition 2. Let $n \in \mathbb{N}$ and $f:[0,1]^{n} \rightarrow[0,1]$. We say that $f$ is weakly increasing if for all $\mathbf{x} \in[0,1]^{n}$ and $c>0$ such that $\mathbf{x}+c \mathbf{1} \in[0,1]^{n}$, it holds that $f(\mathbf{x}) \leq f(\mathbf{x}+c \mathbf{1})$. Similarly, $f$ is weakly decreasing if for all $\mathbf{x} \in[0,1]^{n}$ and $c>0$ such that $\mathbf{x}+c \mathbf{1} \in$ $[0,1]^{n}$, it holds that $f(\mathbf{x}) \geq f(\mathbf{x}+c \mathbf{1})$.

If a function $f$ is both weakly increasing and weakly decreasing, then $f$ is said to be weakly constant.

This concept was extended considering an arbitrary direction $\vec{r} \in \mathbb{R}^{n}$ instead of the vector 1, leading to the notion of directional monotonicity [8].

Definition 3. Let $n \in \mathbb{N}, \vec{r} \in \mathbb{R}^{n} \backslash\{\overrightarrow{0}\}$ and $f:[0,1]^{n} \rightarrow[0,1]$. We say that $f$ is $\vec{r}$-increasing, if for all $c>0$ and $\mathbf{x} \in[0,1]^{n}$ such that $\mathbf{x}+c \vec{r} \in[0,1]^{n}$, it holds that $f(\mathbf{x}) \leq f(\mathbf{x}+c \vec{r})$. Similarly, $f$ is $\vec{r}$-decreasing, if for all $c>0$ and $\mathbf{x} \in[0,1]^{n}$ such that $\mathbf{x}+c \vec{r} \in[0,1]^{n}$, it holds that $f(\mathbf{x}) \geq f(\mathbf{x}+c \vec{r})$. 
If a function $f$ is both $\vec{r}$-increasing and $\vec{r}$-decreasing, then $f$ is said to be $\vec{r}$ constant.

The concepts given in Definitions 2 and 3 require that a function satisfies a property of monotonicity along a ray which remains the same for all $\mathbf{x} \in[0,1]^{n}$. The following two relaxed forms of monotonicity are related to directions in $\mathbb{R}^{n}$ as well, but the direction of the monotonicity property changes from certain points of the domain to others.

The next relaxed monotonicity form is known as ordered directional monotonicity, or OD monotonicity for short, [6].

Definition 4. Let $n \in \mathbb{N}, \vec{r} \in \mathbb{R}^{n} \backslash\{\overrightarrow{0}\}$ and $f:[0,1]^{n} \rightarrow[0,1]$. We say that $f$ is ordered directionally $(O D) \vec{r}$-increasing if for all $c>0, \sigma \in \mathscr{S}_{n}$ and $\mathbf{x} \in[0,1]^{n}$ with $x_{\sigma(1)} \geq \cdots \geq x_{\sigma(n)}$ such that

$$
1 \geq x_{\sigma(1)}+c r_{1} \geq \cdots \geq x_{\sigma(n)}+c r_{n} \geq 0,
$$

it holds that

$$
f(\mathbf{x}) \leq f\left(\mathbf{x}+c \vec{r}_{\sigma^{-1}}\right),
$$

where $\sigma^{-1}$ is the inverse permutation of $\sigma$. Similarly, we say that $f$ is $O D \vec{r}$ decreasing if for all $c>0, \sigma \in \mathscr{S}_{n}$ and $\mathbf{x} \in[0,1]^{n}$ with $x_{\sigma(1)} \geq \cdots \geq x_{\sigma(n)}$ such that

$$
1 \geq x_{\sigma(1)}+c r_{1} \geq \cdots \geq x_{\sigma(n)}+c r_{n} \geq 0
$$

it holds that

$$
f(\mathbf{x}) \geq f\left(\mathbf{x}+c \vec{r}_{\sigma^{-1}}\right) .
$$

If a function $f$ is both OD $\vec{r}$-increasing and OD $\vec{r}$-decreasing, then $f$ is said to be OD $\vec{r}$-constant.

Based on the concept of OD monotonicity, in [19] the concept of strengthened ordered directional monotonicity was introduced.

Definition 5. Let $n \in \mathbb{N}, \vec{r} \in \mathbb{R}^{n} \backslash\{\overrightarrow{0}\}$ and $f:[0,1]^{n} \rightarrow[0,1]$. We say that $f$ is strengthened ordered directionally (SOD) $\vec{r}$-increasing if for all $c>0, \sigma \in \mathscr{S}_{n}$ and $\mathbf{x} \in[0,1]^{n}$ with $x_{\sigma(1)} \geq \cdots \geq x_{\sigma(n)}$ such that $\mathbf{x}_{\sigma}+c \vec{r} \in[0,1]^{n}$, it holds that

$$
f(\mathbf{x}) \leq f\left(\mathbf{x}+c \vec{r}_{\sigma^{-1}}\right) .
$$

Similarly, we say that $f$ is $S O D \vec{r}$-decreasing if for all $c>0, \sigma \in \mathscr{S}_{n}$ and $\mathbf{x} \in[0,1]^{n}$ with $x_{\sigma(1)} \geq \cdots \geq x_{\sigma(n)}$ such that $\mathbf{x}_{\sigma}+c \vec{r} \in[0,1]^{n}$, it holds that

$$
f(\mathbf{x}) \geq f\left(\mathbf{x}+c \vec{r}_{\sigma^{-1}}\right) .
$$

If a function $f$ is both SOD $\vec{r}$-increasing and SOD $\vec{r}$-decreasing, then $f$ is said to be SOD $\vec{r}$-constant.

Example 1. Let $L:[0,1]^{2} \rightarrow[0,1]$ be the Lehmer mean, i.e., the function given by 


$$
L(x, y)=\frac{x^{2}+y^{2}}{x+y}
$$

with the convention $\frac{0}{0}=0$. This function is weakly increasing. In fact, $L$ only increases along the direction given by the vector $(1,1)[8]$.

Example 2. Let $f:[0,1]^{2} \rightarrow[0,1]$ be the function given by

$$
f(x, y)=|x-y| .
$$

Function $f$ is SOD $\vec{r}$-increasing for all $\vec{r}=\left(r_{1}, r_{2}\right) \in \mathbb{R}^{2} \backslash\{(0,0)\}$ such that $r_{1} \geq$ $r_{2}$.

Indeed, let $\mathbf{x} \in[0,1]^{2}, \sigma \in \mathscr{S}_{2}$ and $c>0$ such that $x_{\sigma(1)} \geq x_{\sigma(2)}$ and $\mathbf{x}+c \vec{r}_{\sigma^{-1}} \in$ $[0,1]^{2}$. For the case $x_{1} \geq x_{2}$, clearly $\sigma=\left(\begin{array}{ll}1 & 2\end{array}\right)$ and since $r_{1} \geq r_{2}$, it holds that

$$
f(x, y)=|x-y| \leq|x-y|+c r_{1}-c r_{2}=\left|x+c r_{1}-y-c r_{2}\right|=f\left(x_{1}+c r_{1}, x_{2}+c r_{2}\right) .
$$

The case in which $x_{1} \leq x_{2}$ is similar taking into account that $\sigma=(21)$.

\subsection{Properties}

The direction given by a vector $\vec{r} \in \mathbb{R}^{n} \backslash\{\overrightarrow{0}\}$ and the one given by any other vector $\alpha \vec{r}$ for any $\alpha>0$ coincide. In consequence, for all the monotonicity conditions with which we deal, it is equivalent to increase along $\vec{r}$ and along $\alpha \vec{r}$ for any $\alpha>0$.

Proposition 1. Let $\vec{r} \in \mathbb{R}^{n} \backslash\{\overrightarrow{0}\}, f:[0,1]^{n} \rightarrow[0,1]$ and $\alpha>0$. Then,

1. $f$ is weakly increasing if and only if $f$ is $(\alpha \overrightarrow{1})$-increasing;

2. $f$ is $\vec{r}$-increasing if and only if $f$ is $(\alpha \vec{r})$-increasing;

3. $f$ is $O D \vec{r}$-increasing if and only if $f$ is $O D(\alpha \vec{r})$-increasing;

4. $f$ is $S O D \vec{r}$-increasing if and only if $f$ is $S O D(\alpha \vec{r})$-increasing.

By Proposition 1, the norm of a vector $\vec{r}$ has no effect in any of the conditions of monotonicity. Therefore, we can find a unique representative vector for each direction by requiring that $\|\vec{r}\|=1$.

Moreover, from the definitions, it is clear that weak monotonicity is a particular case of directional monotonicity and, due to the restriction (1), it is also clear that if a function $f$ is SOD $\vec{r}$-monotone for some $\vec{r} \in \mathbb{R}^{n} \backslash\{\overrightarrow{0}\}$, then $f$ is also OD $\vec{r}$ monotone. The converse implication is not true [19]. However, if the components of $\vec{r}$ are decreasingly ordered, then the concepts of OD and SOD monotonicity are equivalent.

Proposition 2. Let $f:[0,1]^{n} \rightarrow[0,1]$ and let $\vec{r} \in \mathbb{R}^{n} \backslash\{\overrightarrow{0}\}$ such that $r_{1} \geq \ldots \geq r_{n}$. Function $f$ is $O D \vec{r}$-increasing if and only if it is SOD $\vec{r}$-increasing. 
Obviously, Propositions 1 and 2 can be equivalently stated in terms of OD and SOD $\vec{r}$-decreasingness and $\vec{r}$-constantness.

The next result shows another difference between OD monotone functions and SOD monotone functions.

Proposition 3. Let $\vec{r} \in \mathbb{R}^{n} \backslash\{\overrightarrow{0}\}$. Then,

1. A function $f:[0,1]^{n} \rightarrow[0,1]$ is $\vec{r}$-increasing if and only if it is $(-\vec{r})$ decreasing;

2. A function $f:[0,1]^{n} \rightarrow[0,1]$ is $O D \vec{r}$-increasing if and only if it is $O D(-\vec{r})$ decreasing.

Proposition 3 does not hold for SOD monotone functions. In fact, in Section 3.1 we present an example (Example 2) of a function that is SOD $\vec{r}$-increasing but not $\operatorname{SOD}(-\vec{r})$-decreasing.

A relevant property that is satisfied by any function that meets one of the discussed monotonicity criteria is that if it increases along two directions, then it increases along the positive convex combination of those directions. The following three results state this fact for each concept of monotonicity.

Theorem 1 ([|]|). Let $\vec{r}, \vec{s} \in \mathbb{R}^{n} \backslash\{\overrightarrow{0}\}$ and $a, b>0$. Let $\mathbf{x} \in[0,1]^{n}$ and $c>0$ such that if $\mathbf{x}$ and $\mathbf{x}+c(a \vec{r}+b \vec{s}) \in[0,1]^{n}$, then $\mathbf{x}+c a \vec{r} \in[0,1]^{n}$ or $\mathbf{x}+c b \vec{s} \in[0,1]^{n}$. Then, if a function $f:[0,1]^{n} \rightarrow[0,1]$ is $\vec{r}$-increasing and $\vec{s}$-increasing, it is also $(a \vec{r}+b \vec{s})$-increasing.

Theorem 2 ([б]). Let $\vec{r}, \vec{s} \in \mathbb{R}^{n} \backslash\{\overrightarrow{0}\}$ and $a, b>0$. Let $\mathbf{x} \in[0,1]^{n}, c>0$ and $\sigma \in \mathscr{S}_{n}$ such that if $1 \geq x_{\sigma(1)} \geq \ldots \geq x_{\sigma(n)} \geq 0$ and

$$
1 \geq x_{\sigma(1)}+c\left(a r_{1}+b s_{1}\right) \geq \ldots \geq x_{\sigma(n)}+c\left(a r_{n}+b s_{n}\right) \geq 0,
$$

then either

$$
1 \geq x_{\sigma(1)}+\operatorname{car}_{1} \geq \ldots \geq x_{\sigma(n)}+c a r_{n} \geq 0
$$

or

$$
1 \geq x_{\sigma(1)}+c b s_{1} \geq \ldots \geq x_{\sigma(n)}+c b s_{n} \geq 0 .
$$

Then, if a function $f:[0,1]^{n} \rightarrow[0,1]$ is $O D \vec{r}$-increasing and $O D \vec{s}$-increasing, it is also OD $(a \vec{r}+b \vec{s})$-increasing.

Theorem 3 ([19]). Let $\vec{r}, \vec{s} \in \mathbb{R}^{n}$ and $a, b>0$. Let $\mathbf{x} \in[0,1]^{n}, c>0$ and $\sigma \in \mathscr{S}_{n}$ such that if $1 \geq x_{\sigma(1)} \geq \ldots \geq x_{\sigma(n)} \geq 0$ and $\mathbf{x}_{\sigma}+c(a \vec{r}+b \vec{s}) \in[0,1]^{n}$, then either $\mathbf{x}+c a \vec{r} \in[0,1]^{n}$ or $\mathbf{x}+c b \vec{s} \in[0,1]^{n}$. Then, if a function $f:[0,1]^{n} \rightarrow[0,1]$ is SOD $\vec{r}$-increasing and SOD $\vec{s}$-increasing, it is also SOD $(a \vec{r}+b \vec{s})$-increasing.

As a consequence of Theorems 1, 2 and 3, it is equivalent for a function to increase, in the standard sense, and to increase, in the sense of the three notions of monotonicity, with respect a set of vectors that span the set of all positive vectors. 
Theorem 4 ([19]). Let $f:[0,1]^{n} \rightarrow[0,1]$ and $\left(\vec{e}_{1}, \ldots, \vec{e}_{n}\right)$ be the canonical basis of $\mathbb{R}^{n}$, i.e., the set of vectors such that $\vec{e}_{i}=\left(0, \ldots, 0, \frac{1}{\hat{i}}, 0, \ldots, 0\right) \in \mathbb{R}^{n}$ for each $i \in\{1, \ldots, n\}$. Then, the following statements are equivalent:

1. $f$ is increasing;

2. $f$ is $\vec{e}_{i}$-increasing for all $i \in\{1, \ldots, n\}$;

3. $f$ is $O D \vec{e}_{i}$-increasing for all $i \in\{1, \ldots, n\}$;

4. $f$ is $S O D \vec{e}_{i}$-increasing for all $i \in\{1, \ldots, n\}$.

Note that for all $\sigma \in \mathscr{S}_{n}$ it holds that $\overrightarrow{1}_{\sigma^{-1}}=\overrightarrow{1}$ and, hence, it is straight that for a function $f:[0,1]^{n} \rightarrow[0,1]$ it is equivalent to be weakly increasing, OD $\overrightarrow{1}$ increasing and SOD $\overrightarrow{1}$-increasing.

\section{Local study of the different notions of monotonicity}

The aforementioned conditions of monotonicity are global properties, in the sense that they require to be fulfilled for all the points in the domain $[0,1]^{n}$. In [17], the local notion of pointwise directional monotonicity was introduced.

Definition 6. Let $\vec{r} \in \mathbb{R}^{n} \backslash\{\overrightarrow{0}\}$ and $f:[0,1]^{n} \rightarrow[0,1]$. We say that $f$ is $\vec{r}$ increasing at $\mathbf{x} \in[0,1]^{n}$ if for all $c>0$ such that $\mathbf{x}+c \vec{r} \in[0,1]^{n}$, it holds that $f(\mathbf{x}) \leq f(\mathbf{x}+c \vec{r})$. Similarly, $f$ is $\vec{r}$-decreasing at $\mathbf{x} \in[0,1]^{n}$ if for all $c>0$ such that $\mathbf{x}+c \vec{r} \in[0,1]^{n}$, it holds that $f(\mathbf{x}) \geq f(\mathbf{x}+c \vec{r})$.

Thus, weak and directional monotonicity can be characterized by means of pointwise directional monotonicity. Clearly, given $\vec{r} \in \mathbb{R}^{n} \backslash\{\overrightarrow{0}\}$, it is equivalent for a function $f:[0,1]^{n} \rightarrow[0,1]$ to be $\vec{r}$-increasing and to be $\vec{r}$-increasing at $\mathbf{x}$, for all $\mathbf{x} \in[0,1]^{n}$. The case of weak monotonicity follows similarly considering the vector $\vec{r}=\overrightarrow{1}$.

Remark 1. Note that the property of directional monotonicity stated in Proposition 3 does not hold for pointwise directional monotonicity at a specific point. Namely, if a function $f:[0,1]^{n} \rightarrow[0,1]$ is $\vec{r}$-increasing at $\mathbf{x} \in[0,1]^{n}$ for some vector $\vec{r} \in$ $\mathbb{R}^{n} \backslash\{\overrightarrow{0}\}$, it does not necessarily hold that $f$ is $(-\vec{r})$-decreasing at $\mathbf{x}$.

Indeed, let $f:[0,1]^{2} \rightarrow[0,1]$ be the function given by

$$
f(x, y)=2 x^{2}+2 y^{2}-2 x-2 y+1 .
$$

One can easily verify that function $f$ has a global minimum at the point $(0.5,0.5)$, in which $f(0.5,0.5)=0$, and, also, that $f(x, y)>0$ for all $(x, y) \in[0,1]^{2} \backslash\{(0,0)\}$. Hence, $f$ is $\vec{r}$-increasing at $(0.5,0.5)$ for every possible $\vec{r} \in \mathbb{R}^{n} \backslash\{\overrightarrow{0}\}$. In particular, given $\vec{r} \in \mathbb{R}^{n} \backslash\{\overrightarrow{0}\}, f$ is $\vec{r}$-increasing and $(-\vec{r})$-increasing. However, $f$ is not $\vec{r}$-decreasing with respect to any direction $\vec{r} \in \mathbb{R}^{n} \backslash\{\overrightarrow{0}\}$. 
As for weak monotonicity and directional monotonicity, it is possible to characterize the concepts of OD monotonicity and SOD monotonicity in terms of pointwise directional monotonicity. For that, we need to introduce first some specific subsets of $[0,1]^{n}$.

Let $\sigma \in \mathscr{S}_{n}$ and let us set $\Omega_{\sigma} \subset[0,1]^{n}$ as follows:

$$
\Omega_{\sigma}=\left\{\mathbf{x} \in[0,1]^{n} \mid x_{\sigma(1)} \geq x_{\sigma(2)} \geq \ldots \geq x_{\sigma(n)}\right\} .
$$

Thus, $\Omega_{\sigma}$ is the set of points $\mathbf{x} \in[0,1]^{n}$ such that $\mathbf{x}_{\sigma}$ is decreasingly ordered.

Let us first start with the characterization of SOD monotonicity in terms of pointwise directional monotonicity.

Theorem 5. Let $\vec{r} \in \mathbb{R}^{n} \backslash\{\overrightarrow{0}\}$. A function $f:[0,1]^{n} \rightarrow[0,1]$ is SOD $\vec{r}$-increasing

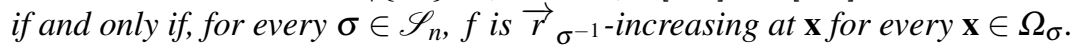

Proof. Let $f$ be SOD $\vec{r}$-increasing. If $\mathbf{x} \in[0,1]^{n}$ and $\sigma \in \mathscr{S}_{n}$ such that $x_{\sigma(1)} \geq \ldots \geq$ $x_{\sigma(n)}$, then $\mathbf{x} \in \Omega_{\sigma}$. Since $f$ is SOD $\vec{r}$-increasing, then by Definition 5 , it holds that

$$
f(\mathbf{x}) \leq f\left(\mathbf{x}+c \vec{r}_{\sigma^{-1}}\right),
$$

for some $c>0$. Hence, $f$ is $\vec{r}_{\sigma^{-1}}$-increasing at $\mathbf{x}$.

Conversely, let $f$ be $\vec{r} \sigma^{-1}$-increasing at $\mathbf{x}$ for every $\mathbf{x} \in \Omega_{\sigma}$, for every $\sigma \in \mathscr{S}_{n}$. Thus, if $\mathbf{x} \in[0,1]^{n}$ and $\sigma \in \mathscr{S}_{n}$ such that $x_{\sigma(1)} \geq \ldots \geq x_{\sigma(n)}$, by the $\vec{r}_{\sigma^{-1}-}$ increasingness of $f$ at $\mathbf{x}$, it is clear that $f(\mathbf{x}) \leq f\left(\mathbf{x}+c \vec{r}_{\sigma^{-1}}\right)$, and, therefore, $f$ is SOD $\vec{r}$-increasing.

The preceding result, Theorem 5 cannot be straightforwardly translated to OD monotonicity. In fact, in Example 3 we can find an OD $\vec{r}$-increasing function that is not $\vec{r} \sigma^{-1}$-increasing at $\mathbf{x}$ for all $\mathbf{x} \in \Omega_{\sigma}$. It suffices to consider a vector $\vec{r} \in$ $\mathbb{R}^{2} \backslash\{\overrightarrow{0}\}$ such that $r_{1}<r_{2}$.

Nevertheless, it is true that the converse implication holds naturally for OD monotone functions.

Corollary 1. Let $\vec{r} \in \mathbb{R}^{n} \backslash\{\overrightarrow{0}\}$. If, for every $\sigma \in \mathscr{S}_{n}$, a function $f:[0,1]^{n} \rightarrow[0,1]$ is $\vec{r}_{\sigma^{-1}}$-increasing at $\mathbf{x}$ for every $\mathbf{x} \in \Omega_{\sigma}$, then $f$ is $O D \vec{r}$-increasing.

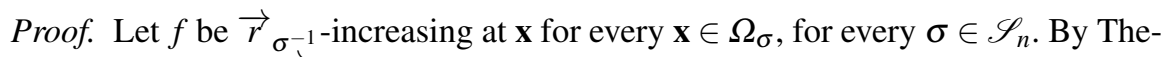
orem 5, $f$ is SOD $\vec{r}$-increasing and, since SOD monotonicity implies OD monotonicity, $f$ is OD $\vec{r}$-increasing.

Corollary 1 fails to be a characterization because OD monotonicity does not require monotonicity conditions for points $\mathbf{x} \in \Omega_{\sigma}$ such that $\mathbf{x}+c \vec{r}_{\sigma^{-1}} \notin \Omega_{\sigma}$, as it means that condition (1) in Definition 4 is not satisfied.

The next result shows the modifications that need to be done in order to characterize OD monotonicity in terms of pointwise directional monotonicity.

Theorem 6. Let $\vec{r} \in \mathbb{R}^{n} \backslash\{\overrightarrow{0}\}$. A function $f:[0,1]^{n} \rightarrow[0,1]$ is OD $\vec{r}$-increasing if and only if, for every $\sigma \in \mathscr{S}_{n}$, the restricted function $\left.f\right|_{\Omega_{\sigma}}:\left(\Omega_{\sigma}\right)^{n} \rightarrow[0,1]$ is

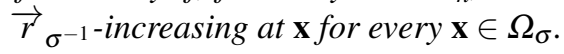


Proof. Let $f$ be OD $\vec{r}$-increasing and let $c>0, \sigma \in \mathscr{S}_{n}$ and $\mathbf{x} \in[0,1]^{n}$ such that $x_{\sigma(1)} \geq \cdots \geq x_{\sigma(n)}$ and

$$
1 \geq x_{\sigma(1)}+c r_{1} \geq \cdots \geq x_{\sigma(n)}+c r_{n} \geq 0 .
$$

Then, $\mathbf{x} \in \Omega_{\sigma}$ and $\mathbf{x}+c \vec{r}_{\sigma^{-1}} \in \Omega_{\sigma}$. Therefore, $f(\mathbf{x})=\left.f\right|_{\Omega_{\sigma}}(\mathbf{x})$ and $f\left(\mathbf{x}+c \vec{r}_{\sigma^{-1}}\right)=$ $\left.f\right|_{\Omega_{\sigma}}\left(\mathbf{x}+c \vec{r} \sigma_{\sigma^{-1}}\right)$. Thus, since $f$ is OD $\vec{r}$-increasing, it holds that

$$
\left.f\right|_{\Omega_{\sigma}}(\mathbf{x})=f(\mathbf{x}) \leq f\left(\mathbf{x}+c \vec{r} \sigma_{\sigma^{-1}}\right)=\left.f\right|_{\Omega_{\sigma}}\left(\mathbf{x}+c \vec{r}_{\sigma^{-1}}\right) .
$$

The converse implication follows analogously.

The next is an example that remarks the differences between SOD and OD monotonicity from a local point of view. The function in it is a restricted equivalence function (REF) [5], which have been used for diverse applications [11, 18].

Example 3. If we consider the function $R E F:[0,1]^{2} \rightarrow[0,1]$ given by

$$
R E F(x, y)=1-|x-y| .
$$

To study the pointwise directional monotonicity of $R E F$, let us firt note that for a vector $\vec{r}=(r, r) \in \mathbb{R}^{2} \backslash\{(0,0)\}$ it holds that $R E F(x, y)=R E F(x+c r, y+c r)$ for every $(x, y) \in[0,1]^{2}$. Therefore, $R E F$ is $(r, r)$-constant for all $0 \neq r \in \mathbb{R}$. Now, if $\vec{r}=\left(r_{1}, r_{2}\right) \in \mathbb{R}^{2} \backslash\{(0,0)\}$ is such that $r_{1} \neq r_{2}$, note that

$$
\operatorname{REF}(0.8,0.8)=1>1-\left|c r_{1}-c r_{2}\right|=\operatorname{REF}\left(0.8+c r_{1}, 0.8+c r_{2}\right) .
$$

Thus, REF is not $\vec{r}$-increasing at $(0.8,0.8)$ for any $\vec{r}$ such that $r_{1} \neq r_{2}$. Consequently, by the characterization of global directional montonicity in terms of pointwise directional monotonicity, REF is $\left(r_{1}, r_{2}\right)$-increasing if and only if $r_{1}=r_{2} \neq 0$.

Let us proceed to study OD and SOD monotonicity of $R E F$. In the 2-dimensional case, there exist two possible permutations $\sigma_{1}=\left(\begin{array}{ll}1 & 2\end{array}\right)$ and $\sigma_{2}=\left(\begin{array}{ll}2 & 1\end{array}\right) \in \mathscr{S}_{2}$, whose respective inverse coincides with themselves and which define the following subsets:

$$
\begin{aligned}
& \Omega_{\sigma_{1}}=\left\{\mathbf{x} \in[0,1]^{2} \mid x_{1} \geq x_{2}\right\}, \text { and } \\
& \Omega_{\sigma_{2}}=\left\{\mathbf{x} \in[0,1]^{2} \mid x_{1} \leq x_{2}\right\} .
\end{aligned}
$$

Clearly, by the same argument as before, $R E F$ is OD and SOD $(r, r)$-increasing for all $0 \neq r \in \mathbb{R}$. Hence, we can focus on the case of $\vec{r}=\left(r_{1}, r_{2}\right) \in \mathbb{R}^{2} \backslash\{(0,0)\}$ such that $r_{1} \neq r_{2}$.

On the one hand, if $r_{1}>r_{2}$, let $(0.8,0.2) \in[0,1]^{2}$. It holds that $(0.8,0.2) \in \Omega_{\sigma_{1}}$ and there exists $c>0$ such that $\left(0.8+c r_{1}, 0.2+c r_{2}\right) \in \Omega_{\sigma_{1}}$ and, hence,

$$
\operatorname{REF}(0.8,0.2)=0.4>1-\left|0.6+c\left(r_{1}-r_{2}\right)\right|=R E F\left(0.8+c r_{1}, 0.2+c r_{2}\right) .
$$

Therefore, by Theorems 5 and $6, R E F$ is neither OD, nor SOD $\left(r_{1}, r_{2}\right)$-increasing for $\vec{r} \in \mathbb{R}^{2} \backslash\{(0,0)\}$ such that $r_{1}>r_{2}$. 
On the other hand, if $r_{1}<r_{2}$, first let $(0.8,0.8) \in \Omega_{\sigma_{1}}$. Thus, there exists $c>0$ such that $\left(0.8+c r_{1}, 0.8+c r_{2}\right) \in \Omega_{2}$ and

$$
\operatorname{REF}(0.8,0.8)=1>1-c\left(r_{2}-r_{1}\right)=\operatorname{REF}\left(0.8+c r_{1}, 0.8+c r_{2}\right) .
$$

Consequently, by Theorem $5, R E F$ is not SOD $\vec{r}$-increasing.

However, it is easy to check that in the cases that $(x, y),(x, y)+c\left(r_{1}, r_{2}\right) \in \Omega_{\sigma_{1}}$ and $(x, y),(x, y)+c\left(r_{2}, r_{1}\right) \in \Omega_{\sigma_{2}}$, the conditions $R E F(x, y) \leq R E F\left(x+c r_{1}, y+c r_{2}\right)$ and $R E F(x, y) \leq R E F\left(x+c r_{2}, y+c r_{1}\right)$, respectively, hold. Therefore, $R E F$ is OD $\vec{r}$-increasing.

In conclusion, $R E F$ is SOD $\vec{r}$-increasing if and only if $\vec{r}=(r, r) \in \mathbb{R}^{2} \backslash\{(0,0)\}$ and REF is OD $\vec{r}$-increasing if and only if $\vec{r}=\left(r_{1}, r_{2}\right) \in \mathbb{R}^{2} \backslash\{(0,0)\}$ such that $r_{1} \leq r_{2}$.

\section{Conclusions}

We have gone over some of the recently introduced relaxed forms of monotonicity. Particularly, we have discussed the notions of weak, directional, OD and SOD monotonicity. We have also discussed some of the properties that functions verifying the cited monotonicity conditions satisfy and we have carried out a study of the local effect of this monotonicity conditions at specific points of the domain. In that attempt, we have recalled the notion of pointwise directional monotonicity and we have used it to characterize these relaxations. Additionally, we have pointed out some differences between OD and SOD monotonicity by means of an instance of a restricted equivalence function.

\section{Acknowledgments}

This work is supported by the project TIN2016-77356-P (AEI/FEDER, UE), by the Public University of Navarra under the project PJUPNA13 and by Slovak grant APVV-14-0013.

\section{References}

1. Bedregal, B., Reiser, R., Bustince, H., Lopez-Molina, C., Torra, V.: Aggregation functions for typical hesitant fuzzy elements and the action of automorphisms. Information Sciences 255, 82-99 (2014)

2. Beliakov, G., Bustince, H., Calvo, T.: A Practical Guide to Averaging Functions. Studies in Fuzziness and Soft Computing. Springer International Publishing (2016)

3. Beliakov, G., Calvo, T., Wilkin, T.: Three types of monotonicity of averaging functions. Knowledge-Based Systems 72, 114-122 (2014). DOI 10.1016/j.knosys.2014.08.028 
4. Beliakov, G., Calvo, T., Wilkin, T.: On the weak monotonicity of Gini means and other mixture functions. Information Sciences 300, 70-84 (2015)

5. Bustince, H., Barrenechea, E., Pagola, M.: Restricted equivalence functions. Fuzzy Sets and Systems 157(17), 2333-2346 (2006). DOI 10.1016/j.fss.2006.03.018

6. Bustince, H., Barrenechea, E., Sesma-Sara, M., Lafuente, J., Dimuro, G.P., Mesiar, R., Kolesárová, A.: Ordered directionally monotone functions. Justification and application. IEEE Transactions on Fuzzy Systems 26(4), 2237-2250 (2018). DOI 10.1109/TFUZZ.2017. 2769486

7. Bustince, H., Fernández, J., Kolesárová, A., Mesiar, R.: Generation of linear orders for intervals by means of aggregation functions. Fuzzy Sets and Systems 220, 69-77 (2013)

8. Bustince, H., Fernandez, J., Kolesárová, A., Mesiar, R.: Directional monotonicity of fusion functions. European Journal of Operational Research 244(1), 300-308 (2015). DOI 10.1016/ j.ejor.2015.01.018

9. De Miguel, L., Sesma-Sara, M., Elkano, M., Asiain, M., Bustince, H.: An algorithm for group decision making using n-dimensional fuzzy sets, admissible orders and OWA operators. Information Fusion 37, 126-131 (2017). DOI 10.1016/j.inffus.2017.01.007

10. Deckỳ, M., Mesiar, R., Stupňanová, A.: Deviation-based aggregation functions. Fuzzy Sets and Systems 332, 29-36 (2018)

11. Galar, M., Fernandez, J., Beliakov, G., Bustince, H.: Interval-valued fuzzy sets applied to stereo matching of color images. IEEE Transactions on Image Processing 20(7), 1949-1961 (2011)

12. Grabisch, M., Marichal, J., Mesiar, R., Pap, E.: Aggregation functions. Cambridge University Press (2009)

13. Lucca, G., Sanz, J., Dimuro, G., Bedregal, B., Asiain, M.J., Elkano, M., Bustince, H.: CCintegrals: Choquet-like copula-based aggregation functions and its application in fuzzy rulebased classification systems. Knowledge-Based Systems 119, 32-43 (2017). DOI 10.1016/j. knosys.2016.12.004

14. Lucca, G., Sanz, J.A., Dimuro, G.P., Bedregal, B., Bustince, H., Mesiar, R.: CF-integrals: A new family of pre-aggregation functions with application to fuzzy rule-based classification systems. Information Sciences 435, 94-110 (2018)

15. Lucca, G., Sanz, J.A., Dimuro, G.P., Bedregal, B., Mesiar, R., Kolesárová, A., Bustince, H.: Preaggregation functions: Construction and an application. IEEE Transactions on Fuzzy Systems 24(2), 260-272 (2016). DOI 10.1109/TFUZZ.2015.2453020

16. Paternain, D., Fernandez, J., Bustince, H., Mesiar, R., Beliakov, G.: Construction of image reduction operators using averaging aggregation functions. Fuzzy Sets and Systems 261, 87111 (2015). DOI 10.1016/j.fss.2014.03.008

17. Sesma-Sara, M., De Miguel, L., Roldán López de Hierro, A.F., Lafuente, J., Mesiar, R., Bustince, H.: Pointwise directional increasingness and geometric interpretation of directionally monotone functions. Information Sciences (Submitted)

18. Sesma-Sara, M., De Miguel, L., Pagola, M., Burusco, A., Mesiar, R., Bustince, H.: New measures for comparing matrices and their application to image processing. Applied Mathematical Modelling 61, 498-520 (2018)

19. Sesma-Sara, M., Lafuente, J., Roldán, A., Mesiar, R., Bustince, H.: Strengthened ordered directionally monotone functions. Links between the different notions of monotonicity. Fuzzy Sets and Systems 357, 151-172 (2019). DOI 10.1016/j.fss.2018.07.007

20. Sesma-Sara, M., Mesiar, R., Bustince, H.: Weak and directional monotonicity of functions on Riesz spaces to fuse uncertain data. Fuzzy Sets and Systems (In press). DOI 10.1016/j.fss. 2019.01.019

21. Wei, G.: Some geometric aggregation functions and their application to dynamic multiple attribute decision making in the intuitionistic fuzzy setting. International Journal of Uncertainty, Fuzziness and Knowledge-Based Systems 17(02), 179-196 (2009)

22. Wilkin, T., Beliakov, G.: Weakly monotonic averaging functions. International Journal of Intelligent Systems 30(2), 144-169 (2015). DOI 10.1002/int.21692 